\title{
A GENERAL RESULT REGARDING THE GROWTH OF SOLUTIONS OF FIRST-ORDER DIFFERENTIAL EQUATIONS ${ }^{1}$
}

\author{
STEVEN B. BANK
}

\begin{abstract}
In this paper, we treat first-order algebraic differential equations whose coefficients are arbitrary complex-valued functions on an interval $\left[x_{0},+\infty\right)$, and we obtain an estimate on the growth of all real-valued solutions on $\left[x_{0},+\infty\right)$. Our result includes, as a very special case, the well-known result of Lindelöf for polynomial coefficients.
\end{abstract}

1. Introduction. In this paper we treat first-order algebraic differential equations, $\Omega\left(x, y, y^{\prime}\right)=0$, where $\Omega\left(x, y, y^{\prime}\right)=\sum f_{i j}(x) y^{i}\left(y^{\prime}\right)^{j}$ is a polynomial in $y$ and $y^{\prime}$ whose coefficients $f_{i j}(x)$ are arbitrary complex-valued functions defined on an interval $\left[x_{0},+\infty\right)$. Our main result ( $\$ 2$ below) provides a growth estimate for any real-valued solution of $\Omega\left(x, y, y^{\prime}\right)=0$ on $\left[x_{0},+\infty\right)$, in terms of a monotone nondecreasing majorant $M(x)$ of all the functions $\left|f_{i j}(x)\right|$, and a positive monotone nonincreasing function $N(x)$ which is majorized by $\left|f_{p-m, m}(x)\right|$, where $p$ is the total degree of $\Omega$ in $y$ and $y^{\prime}$, and where $m$ is the maximum integer $j$ for which the coefficient $f_{p-j, j}(x)$ is not identically zero. (Thus our result applies only to those equations $\Omega\left(x, y, y^{\prime}\right)=$ 0 , for which the "leading" coefficient $f_{p-m, m}(x)$ is nowhere zero on some interval $\left[x_{0},+\infty\right)$.) The estimate is actually obtained in terms of the function $U(x)=(m+1) M(x) / N(x)$, and our result states that if $y(x)$ is a realvalued solution of $\Omega\left(x, y, y^{\prime}\right)=0$ on an interval $\left[x_{0},+\infty\right)$, then,

$$
y(x)=O\left(\exp \left(U(x)+\int_{x_{0}}^{x} U(t) d t\right)\right) \text { as } x \rightarrow+\infty .
$$

(Actually, we obtain a slight improvement of (1) in the case where $|y(x)|$ grows sufficiently rapidly.) It should be pointed out that in the case where $|y(x)|$ does not grow sufficiently rapidly, we require that $U(x)$ be differentiable and have the property that for any $\varepsilon>0$, the function $U^{\prime}(x) / \exp (\varepsilon U(x))$ tends to zero as $x \rightarrow+\infty$. Of course, this property is possessed by the functions which usually serve as majorants namely $\exp _{k}\left(x^{A}\right)$, where $\exp _{k}$ is the $k$ th iterate of the exponential function, and $A$ is a constant. (See also $\$ 6$ below.)

As a very special case of our result, we obviously obtain another proof of

Received by the editors August 10, 1977.

AMS (MOS) subject classifications (1970). Primary $34 \mathrm{Cl0}$.

Key words and phrases. Algebraic differential equations, growth of solutions.

'This research was supported in part by the National Science Foundation (MCS 76-07214). 
Lindelö's theorem [6] which states that if all the coefficients $f_{i j}(x)$ are polynomials, then any real-valued solution $y(x)$ on an interval $\left[x_{0},+\infty\right)$ satisfies an inequality of the form, $|y(x)| \leqslant \exp \left(x^{A}\right)$ for all sufficiently large $x$, where $A$ is a constant. However, we emphasize that our result will provide a growth estimate for the real-valued solutions of any first-order algebraic differential equation for which we possess the upper and lower estimates $M(x)$ and $N(x)$.

We conclude with three remarks. First, no analogue of our result can hold for either complex-valued solutions of first-order algebraic differential equations or real-valued solutions of higher order algebraic differential equations, since such solutions of such equations (even with constant coefficients) can dominate any preassigned function on $(0,+\infty)$ at a sequence of $x$ tending to $+\infty$. (See Vijayaraghavan [9] and Vijayaraghavan, Basu and Bose [10]. In addition, we mention that the construction in [10] was extended to show $[1, \S 14$, p. 53] that increasing solutions of third-order algebraic differential equations with constant coefficients can also have arbitrarily rapid growth.) Secondly, for a survey of the classical results on the growth of real-valued solutions of algebraic differential equations, we refer the reader to Chapter 5 of R. Bellman's book [2]. Finally, for the reader who is interested in the question of growth of real-valued solutions of algebraic difference equations, algebraic functional equations, and algebraic differential-difference equations, we refer the reader to the papers of $\mathbf{K}$. Cooke [3], [4], O. Lancaster [5], and S. M. Shah [7], [8].

2. We now state our main result.

THEOREM. Let $\Omega\left(x, y, y^{\prime}\right)=\Sigma f_{i j}(x) y^{i}\left(y^{\prime}\right)^{j}$ be a polynomial in $y$ and $y^{\prime}$ whose coefficients $f_{i j}(x)$ are complex-valued functions defined on an interval $\left[x_{0}\right.$, $+\infty)$, and let some coefficient be not identically zero. Let $p=\max \{i+j$ : $\left.f_{i j} \neq 0\right\}$. Let $M(x)$ be a monotone nondecreasing function on $\left[x_{0},+\infty\right)$ such that for each $(i, j)$,

$$
\left|f_{i j}(x)\right| \leqslant M(x) \text { for all } x \geqslant x_{0} .
$$

Let $m=\max \left\{j: f_{p-j, j} \not 0\right\}$, and assume $f_{p-m, m}(x)$ is nowhere zero on $\left[x_{0}\right.$, $+\infty)$. Assume further that there exists a monotone nonincreasing function $N(x)$ on $\left[x_{0},+\infty\right)$ such that

$$
\left|f_{p-m, m}(x)\right| \geqslant N(x)>0 \text { for all } x \geqslant x_{0} .
$$

Set $U(x)=(m+1) M(x) / N(x)$. Let $y(x)$ be a real-valued function on $\left[x_{0},+\infty\right)$ which has a continuous first derivative and which satisfies $\Omega(x, y(x)$, $\left.y^{\prime}(x)\right) \equiv 0$ on $\left[x_{0},+\infty\right)$. Then:

(a) If for each $\delta$ in $(0,1)$, the function $N(x)|y(x)|^{\delta} / M(x)$ tends to $+\infty$ as $x \rightarrow+\infty$, then

$$
y(x)=O\left(\exp \left(\int_{x_{0}}^{x} U(t) d t\right)\right) \text { as } x \rightarrow+\infty .
$$


(b) Suppose that for some $\delta$ in $(0,1)$, the function $N(x)|y(x)|^{\delta} / M(x)$ does not tend to $+\infty$ as $x \rightarrow+\infty$. In addition, assume that $U(x)$ is differentiable and satisfies the condition that for any $\varepsilon>0$, the function $U^{\prime}(x) / \exp (\varepsilon U(x))$ tends to zero as $x \rightarrow+\infty$. Then,

$$
y(x)=o\left(\exp \left(U(x)+\int_{x_{0}}^{x} U(t) d t\right)\right) \text { as } x \rightarrow+\infty .
$$

3. We first establish some notation, and then we will prove parts (a) and (b) separately. By dividing the relation $\Omega\left(x, y(x), y^{\prime}(x)\right)=0$ through by $(y(x))^{p}$ (at any point where $y(x) \neq 0$ ), we obtain,

$$
\Lambda(x)=\Phi(x)
$$

where

$$
\begin{aligned}
& \Lambda(x)=\sum_{j=0}^{m} f_{p-j, j}(x)\left(y^{\prime}(x) / y(x)\right)^{j}, \\
& \Phi(x)=-\sum_{i+j<p} h_{i j}(x),
\end{aligned}
$$

and where,

$$
h_{i j}(x)=f_{i j}(x)\left(y^{\prime}(x) / y(x)\right)^{j}(y(x))^{i+j-p}, \text { for } i+j<p .
$$

From (7), we can write,

$$
\Lambda(x)=f_{p-m, m}(x)\left(y^{\prime}(x) / y(x)\right)^{m}\left(1+\sum_{j=0}^{m-1} \Psi_{j}(x)\right),
$$

where for $j=0,1, \ldots, m-1$,

$$
\Psi_{j}(x)=\left(f_{p-j, j}(x) / f_{p-m, m}(x)\right)\left(y^{\prime}(x) / y(x)\right)^{j-m} .
$$

4. Proof of part (a). In this part, we are assuming,

$$
N(x)|y(x)|^{\delta} / M(x) \rightarrow+\infty \quad \text { as } x \rightarrow+\infty \text { for each } \delta \text { in }(0,1) \text {. }
$$

Thus it follows that for all sufficiently large $x$, the function $y(x)$ is either always positive or always negative. We can assume the positive case or otherwise we can apply the argument to $-y$. Hence since $M(x) \geqslant N(x)$, we can assume from (12) that

$$
y(x) \rightarrow+\infty \text { as } x \rightarrow+\infty .
$$

We now assert that for any $\varepsilon>0$, there exists a sequence $\left\{s_{n}\right\} \rightarrow+\infty$ such that,

$$
\left|y^{\prime}\left(s_{n}\right)\right| \leqslant\left(y\left(s_{n}\right)\right)^{1+\varepsilon} \text { for each } n .
$$

To prove (14), we assume the contrary. Then the inequality $\left|y^{\prime}\right|>y^{1+\varepsilon}$ would hold on an interval $\left(x_{1},+\infty\right)$. Setting $g=y^{-\varepsilon}$, it would follow that on $\left(x_{1},+\infty\right)$, we would have $\left|g^{\prime}\right|>\varepsilon$ and hence either $g^{\prime}>\varepsilon$ or $g^{\prime}<-\varepsilon$. However, $g^{\prime}>\varepsilon$ leads to $g \rightarrow+\infty$ as $x \rightarrow+\infty$ which contradicts (13). 
Similarly, $g^{\prime}<-\varepsilon$ leads to $g \rightarrow-\infty$ as $x \rightarrow+\infty$ which again contradicts (13), and thus (14) is proved.

We now assert that for any $\varepsilon>0$, we have

$$
\left|y^{\prime}(x)\right| \leqslant y(x)^{1+\varepsilon} \text { for all sufficiently large } x .
$$

To prove (15), we choose $\delta>0$ so small that $\delta<\min \{\varepsilon, 1\}$ and such that,

$$
\delta<(p-(i+j)) /(j+1) \text { if } f_{i j} \neq 0 \text { and } i+j<p .
$$

It clearly suffices (in view of (13)) to show that,

$$
\left|y^{\prime}(x)\right| \leqslant y(x)^{1+\delta} \text { for all sufficiently large } x .
$$

We assume that (17) is false. In view of (14), it follows that there exists a sequence $\left\{t_{n}\right\} \rightarrow+\infty$ at which $\left|y^{\prime}\right|=y^{1+\delta}$. For $i+j<p$, we consider the functions $h_{i j}$ defined in (9). It easily follows from (2), (13) and our choice of $\delta$ in (16), that $\left|h_{i j}\left(t_{n}\right)\right| \leqslant M\left(t_{n}\right)\left(y\left(t_{n}\right)\right)^{-\delta}$ for all sufficiently large $n$, and hence from (8), we obtain,

$$
\left|\Phi\left(t_{n}\right)\right| \leqslant c M\left(t_{n}\right)\left(y\left(t_{n}\right)\right)^{-\delta}
$$

for all sufficiently large $n$, where $c$ is a positive constant. We now estimate $\left|\Lambda\left(t_{n}\right)\right|$ from below, where $\Lambda$ is given by (10). If $m=0$, then from (3) and (18), it would follow since $\Lambda=\Phi$, that $N\left(t_{n}\right)\left|y\left(t_{n}\right)\right|^{\delta} / M\left(t_{n}\right) \leqslant c$ for all sufficiently large $n$, which clearly contradicts (12). Hence $m \geqslant 1$. We consider the functions $\Psi_{j}$ defined in (11) for $j=0,1, \ldots, m-1$. Since $\left|y^{\prime}\right|=y^{1+\delta}$ at the points $t_{n}$, it easily follows from (2), (3) and (13), that for $j=0,1, \ldots, m-1$, we have $\left|\Psi_{j}\right| \leqslant(M / N) y^{-\delta}$ at the points $t_{n}$, and hence from (12), it follows that $\left|\Psi_{j}\left(t_{n}\right)\right| \leqslant(1 /(m+1))$ for all sufficiently large $n$. Thus from (10) and (3), we see that $|\Lambda| \geqslant N y^{\delta m} /(m+1)$ at $t_{n}$ for all sufficiently large $n$, and hence in view of (13), it follows that $\left|\Lambda\left(t_{n}\right)\right| \geqslant N\left(t_{n}\right)$ for all sufficiently large $n$. This, together with (18) and the fact that $\Lambda=\Phi$, yields the same contradiction of (12) as we obtained in the case $m=0$, and this proves (17) and hence (15).

For $i+j<p$, we can now estimate the functions $h_{i j}(x)$ defined in (9). We choose $\delta$ in $(0,1)$ satisfying (16). Now if $j=0$, then in view of (2) and (13), we have $\left|h_{i j}(x)\right| \leqslant M(x)(y(x))^{-\delta}$ for all sufficiently large $x$. If $j>0$, we can obviously write $h_{i j}=f_{i j}\left(y^{\prime} / y^{1+\alpha}\right)^{j} y^{-\delta}$ where $\alpha>\delta>0$. Thus, in view of (2) and (15), we obtain, $\left|h_{i j}(x)\right| \leqslant M(x)(y(x))^{-\delta}$ for all sufficiently large $x$. Hence, from (8), it clearly follows that,

$$
|\Phi(x)| \leqslant c M(x)(y(x))^{-\delta} \text { for all sufficiently large } x,
$$

where $c$ is a constant.

We now estimate $\Lambda(x)$ which is given by (10). We observe first that we must have $m \geqslant 1$, for if $m=0$, then from (3) we would have $|\Lambda(x)| \geqslant N(x)$ for all sufficiently large $x$, and this, together with (19) and the fact that $\Lambda=\Phi$ would clearly contradict (12). Hence, we must have $m \geqslant 1$ if there is a solution satisfying (12). For convenience, let us denote by $B$, the set of all $x \geqslant x_{0}$ for which $\left|y^{\prime}(x) / y(x)\right| \geqslant U(x)$, where $U(x)=(m+1) M(x) / N(x)$. 
Since $U(x) \geqslant 1$, it follows easily from the definition of $\Psi_{j}(x)$ in (11) and the estimates in (2) and (3), that for $j=0,1, \ldots, m-1$,

$$
\left|\Psi_{j}(x)\right| \leqslant(1 /(m+1)) \text { if } x \text { belongs to } B \text {. }
$$

Hence from the representation for $\Lambda(x)$ in (10), together with the estimate (3), we obtain,

$$
|\Lambda(x)| \geqslant N(x)\left|y^{\prime}(x) / y(x)\right|^{m} /(m+1) \text { for } x \text { in } B .
$$

Thus, if $x$ belongs to $B$, then since $m \geqslant 1$, it follows from (21) that $|\Lambda(x)| \geqslant$ $M(x)$. But from (19) and (13), it follows that for all sufficiently large $x$, we have $|\Phi(x)|<M(x)$, and hence since $\Lambda=\Phi$, we must conclude that if $x$ is sufficiently large, then $x$ cannot belong to $B$. Hence there exists $x_{2} \geqslant x_{0}$ such that,

$$
\left|y^{\prime}(x) / y(x)\right|<U(x) \text { for all } x \geqslant x_{2}
$$

The conclusion (4) now follows easily and part (a) is thus proved.

5. Proof of part (b). First, let us define,

$$
L(x)=\exp \left(U(x)+\int_{x_{0}}^{x} U(t) d t\right) \text { for } x \geqslant x_{0} .
$$

From our assumptions about $U(x)$ in this part, it easily follows that $L(x)$ is a positive, increasing, differentiable function such that,

$$
L(x) \rightarrow+\infty \text { as } x \rightarrow+\infty
$$

and such that for any $\varepsilon>0$, we have

$$
L^{\prime} \leqslant L^{1+\varepsilon} \text { for all sufficiently large } x \text {. }
$$

In this part, we are assuming that our solution $y(x)$ has the property that for some $\delta$ in $(0,1)$, the function $N(x)|y(x)|^{\delta} / M(x)$ does not tend to $+\infty$ as $x \rightarrow+\infty$. Hence there exists a constant $K$ and a sequence $\left\{\zeta_{n}\right\} \rightarrow+\infty$ such that $|y| \leqslant(K M / N)^{1 / \delta}$ at the points $\zeta_{n}$. Since $U=(m+1) M / N$, it easily follows from (23) that,

$$
\left|y\left(\zeta_{n}\right)\right|<L\left(\zeta_{n}\right) \text { for all sufficiently large } n \text {. }
$$

We now assert that,

$$
|y(x)| \leqslant L(x) \text { for all sufficiently large } x .
$$

To prove (27), we assume the contrary. Then there is a sequence $\left\{s_{n}\right\} \rightarrow$ $+\infty$ such that,

$$
\left|y\left(s_{n}\right)\right|>L\left(s_{n}\right) \text { for all } n \text {. }
$$

Let $J$ denote the set of all $x>x_{0}$ for which $|y(x)|>L(x)$. In view of (26) and (28), clearly $J$ contains a countable union of disjoint, finite open intervals $\left(a_{n}, b_{n}\right)$ such that $\left\{b_{n}\right\} \rightarrow+\infty$ and $|y(x)|=L(x)$ if $x=a_{n}$ or $x=b_{n}$. Now since $y(x)$ is clearly of constant sign on each interval $\left(a_{n}, b_{n}\right)$, it follows from Rolle's theorem (applied to $y(x) / L(x)$ ), that there is a sequence $\left\{r_{n}\right\} \rightarrow+\infty$ such that, 


$$
y^{\prime}\left(r_{n}\right) / y\left(r_{n}\right)=L^{\prime}\left(r_{n}\right) / L\left(r_{n}\right) \text { and }\left|y\left(r_{n}\right)\right|>L\left(r_{n}\right) .
$$

We now estimate the value at $r_{n}$ of the functions $h_{i j}$ introduced in (9) for $i+j<p$. In view of (29) and (2), clearly at the point $r_{n}$,

$$
\left|h_{i j}\right| \leqslant M\left(L^{\prime} / L\right)^{j} L^{i+j-p} \text {. }
$$

If $j=0$, then clearly (in view of (24)), $\left|h_{i j}\right| \leqslant M L^{-1}$ at $r_{n}$. Now choose a constant $\sigma$ in $(0,1)$ so small that

$$
\sigma<(p-(i+j)) /(j+1) \text { if } f_{i j} \not \equiv 0 \text { and } i+j<p .
$$

Then, if $j>0$, we can write the right hand side of (30) as $M\left(L^{\prime} / L^{1+\alpha}\right)^{j} L^{-\sigma}$ where $\alpha>\sigma>0$, so in view of (25) and (30), we have $\left|h_{i j}\left(r_{n}\right)\right| \leqslant$ $M\left(r_{n}\right)\left(L\left(r_{n}\right)\right)^{-\sigma}$ for all sufficiently large $n$. Hence from (8), we obtain,

$$
\left|\Phi\left(r_{n}\right)\right| \leqslant c M\left(r_{n}\right)\left(L\left(r_{n}\right)\right)^{-\sigma} \text { for all sufficiently large } n,
$$

where $c$ is a constant. We now consider the value at $r_{n}$ of the functions $\Psi_{j}$ introduced in (11) for $j=0,1, \ldots, m-1$. Clearly, from (2), (3) and (29), we have,

$$
\left|\Psi_{j}\left(r_{n}\right)\right| \leqslant\left(M\left(r_{n}\right) / N\left(r_{n}\right)\right)\left(L^{\prime}\left(r_{n}\right) / L\left(r_{n}\right)\right)^{j-m} .
$$

But from (23), $L^{\prime} / L \geqslant U$ where $U=(m+1) M / N$. Hence since $j<m$ in (33), it follows that $\left|\Psi_{j}\left(r_{n}\right)\right| \leqslant(1 /(m+1))$ for all $n$. It thus follows from (3), (10) and (29) that for all $n$,

$$
\left|\Lambda\left(r_{n}\right)\right| \geqslant N\left(r_{n}\right)\left(L^{\prime}\left(r_{n}\right) / L\left(r_{n}\right)\right)^{m} /(m+1) .
$$

If $m=0$, then since $\Lambda=\Phi$, it would easily follow from (32) and (34) that for all sufficiently large $n$, we would have $L\left(r_{n}\right) \leqslant\left(c U\left(r_{n}\right)\right)^{1 / \sigma}$. However, this is clearly impossible since from (23) it easily follows that $L(x) /(U(x))^{1 / \sigma}$ tends to $+\infty$ as $x \rightarrow+\infty$. On the other hand, if $m \geqslant 1$, then since $c L^{-\sigma} \rightarrow 0$ as $x \rightarrow+\infty$, it would follow from (32) and (34) that for all sufficiently large $n$, we have $\left(L^{\prime}\left(r_{n}\right) / L\left(r_{n}\right)\right)^{m}<U\left(r_{n}\right)$. However, this is clearly impossible since from (23), $L^{\prime} / L \geqslant U$ and, of course, $m \geqslant 1$ and $U(x) \geqslant 1$ for all $x$. This proves (27) and hence part (b) is proved.

6. Remark. In part (b) of the theorem, we require that the monotone nondecreasing function $U(x)$ be differentiable and have the property that for any $\varepsilon>0$, the ratio $U^{\prime} / \exp (\varepsilon U)$ tends to zero as $x \rightarrow+\infty$. We mention here the simple fact that for any differentiable, unbounded, monotone nondecreasing function $U$ on an interval $\left[x_{0},+\infty\right)$, and any $\varepsilon>0$, it is easy to see that the ratio $U^{\prime} / \exp (\varepsilon U)$ tends to zero as $x$ tends to $+\infty$ outside a possible exceptional set of finite measure. This follows easily since the convergence of the integral $\int_{x_{0}}^{+\infty}\left(U^{\prime}(t) / U(t)^{1+\varepsilon}\right) d t$ implies that for any $\varepsilon>0$, the inequality $U^{\prime}<U^{1+\varepsilon}$ holds on $\left[x_{0},+\infty\right)$ with the possible exception of a set of finite measure.

\section{BIBLIOGRAPHY}

1. S. Bank, Some results on analytic and meromorphic solutions of algebraic differential equations, Advances in Math. 15 (1975), 41-62. 
2. R. Bellman, Stability theory of differential equations, Dover, New York, 1953.

3. K. Cooke, The rate of increase of real continuous solutions of algebraic differential-difference equations of the first order, Pacific J. Math. 4 (1954), 483-501.

4. The rate of increase of real continuous solutions of certain algebraic functional equations, Trans. Amer. Math. Soc. 92 (1959), 106-124.

5. O. Lancaster, Some results concerning the behavior at infinity of real continuous solutions of algebraic difference equations, Bull. Amer. Math. Soc. 46 (1940), 169-177.

6. E. Lindelöf, Sur la croissance des intégrales des équations différentielles algébrique du premier ordre, Bull. Soc. Math. France 27 (1899), 205-215.

7. S. M. Shah, On real continuous solutions of algebraic difference equations, Bull. Amer. Math. Soc. 53 (1947), 548-558.

8. , On real continuous solutions of algebraic difference equations. II, Proc. Nat. Inst. Sci. India Sect. A. 16 (1950), 11-17.

9. T. Vijayaraghavan, Sur la croissance des fonctions définies par les équations différentielles, C. R. Acad. Sci. Paris 194 (1932), 827-829.

10. T. Vijayaraghavan, N. Basu and S. Bose, A simple example for a theorem of Vijayaraghavan, J. London Math. Soc. 12 (1937), 250-252.

Department of Mathematics, University of Illinois at Urbana-Champaign, Urbana, ILLINOIS 61801 